\title{
A data-driven alternative to the fractional Fokker-Planck equation
}

\author{
Steve Pressé \\ Department of Physics, IUPUI Indianapolis, 420 University Blvd, Indianapolis, \\ IN 46202, USA \\ E-mail: stevenpresse@gmail.com
}

\begin{abstract}
Anomalous diffusion processes are ubiquitous in biology and arise in the transport of proteins, vesicles and other particles. Such anomalously diffusive behavior is attributed to a number of factors within the cell including heterogeneous environments, active transport processes and local trapping/binding. There are a number of microscopic principles - such as power law jump size and/or waiting time distributions - from which the fractional Fokker-Planck equation (FFPE) can be derived and used to provide mechanistic insight into the origins of anomalous diffusion. On the other hand, it is fair to ask if other microscopic principles could also have given rise to the evolution of an observed density profile that appears to be well fit by an FFPE. Here we discuss another possible mechanistic alternative that can give rise to densities like those generated by FFPEs. Rather than to fit a density (or concentration profile) using a solution to the spatial FFPE, we reconstruct the profile generated by an FFPE using a regular FPE with a spatial and time-dependent force. We focus on the special case of the spatial FFPE for superdiffusive processes. This special case is relevant to, for example, active transport in a biological context. We devise a prescription for extracting such forces on synthetically generated data and provide an interpretation to the forces extracted. In particular, the time-dependence of forces could tell us about ATP depletion or changes in the cell's metabolic activity. Modeling anomalous behavior with normal diffusion driven by these effective forces yields an alternative mechanistic picture that, ultimately, could help motivate future experiments.
\end{abstract}

Keywords: dynamics (theory), diffusion, statistical inference

This is the author's manuscript of the article published in final edited form as:

Pressé, S. (2015). A data-driven alternative to the fractional Fokker-Planck equation. Journal of Statistical Mechanics: Theory and Experiment, 2015(7), P07009. http://doi.org/10.1088/1742-5468/2015/07/P07009 


\section{A data-driven alternative to the fractional Fokker-Planck equation}

\section{Introduction}

On the basis of Fick's laws of diffusion, the normal diffusion equation states that the rate of change of a concentration profile at position $x$ is proportional to its local curvature [1]. Thus, according to normal unbiased diffusion, sharp ( $\delta$-function idealized) initial concentration profiles evolve into Gaussian profiles with mean square displacement, $\left\langle x^{2}\right\rangle$, linear in time.

However, many systems exhibit anomalous diffusion. That is, their mean square displacements are non-linear in time or have correlations in time and space that give rise to non-Gaussian profiles [2,3]. In fact, proteins, vesicles and other particles often diffuse anomalously in cells [4-8]. In the cell's environment, anomalous diffusion is attributed to a number of factors including particle diffusion in crowded environments $[4,9,10]$, active transport [5], particle exploration of fractal cellular structures [11], and particle trap binding [6].

Here, we are interested in building a data-inspired mathematical framework to model anomalous diffusion. Our approach will provide alternative insight to modeling anomalous diffusion processes using the traditional fractional Fokker-Planck equation (FFPE) [2].

To introduce our approach, we briefly highlight the type of insight that can be extracted by fitting anomalous data to an FFPE.

FFPEs can be derived starting from more basic principles $[2,12]$ such as Langevin equations [13], fractional Brownian motion [11, 14-16] or continuous time random walks (CTRWs) $[2,17]$. Each approach provides its own unique microscopic insight.

For example, the CTRW approach to deriving the FFPE begins by defining a jump size $(p(\mathbf{x}))$ and dwell time $(\psi(t))$ distribution for each step taken by a random walker. After many steps, the probability, $P(\mathbf{X}, t)$, of observing the random walker at position $\mathbf{X}$ at time $t$ can be expressed using both jump and time distributions valid for the single step [17]. By Laplace transforming the time variable $(t \rightarrow s)$ and Fourier transforming 
the position variable $(\mathbf{x} \rightarrow \mathbf{k})$ the probability $(P(\mathbf{X}, t) \rightarrow \tilde{\hat{P}}(\mathbf{k}, s))$ becomes $[17]$

$$
\tilde{\hat{P}}(\mathbf{k}, s)=\frac{1-\hat{\psi}(s)}{s} \cdot \frac{1}{1-\hat{\psi}(s) \tilde{p}(\mathbf{k})} .
$$

For a jump time distribution with a finite mean, $\tau$, its long time (short $s$ ) limit is $\hat{\psi}(s) \sim 1-\tau s$. Likewise, in the absence of drift and assuming a finite standard deviation, the dwell time distribution in the small $\mathbf{k}$ limit is $\tilde{p}(\mathbf{k}) \sim 1-\sigma^{2} \mathbf{k}^{2}$ [2]. For this special case, equation (1) becomes

$$
\tilde{\hat{P}}(\mathbf{k}, s)=\frac{\tau}{\tau s+\sigma^{2} \mathbf{k}^{2}} .
$$

Re-arranging the above, we find

$$
s \tilde{\hat{P}}(\mathbf{k}, s)-1=-\frac{\mathbf{k}^{2} \sigma^{2}}{\tau} \tilde{\hat{P}}(\mathbf{k}, s)
$$

which is the Laplace-Fourier inverse of the normal diffusion equation

$$
\partial_{t} P(\mathbf{x}, t)=\frac{\sigma^{2}}{\tau} \nabla^{2} P(\mathbf{x}, t)
$$

Superdiffusion is, instead, obtained by introducing a diverging second moment in the jump distribution $\left(\tilde{p}(\mathbf{k}) \sim 1-\phi|\mathbf{k}|^{\alpha}\right.$ where $\left.1 \leqslant \alpha<2\right)$ which yields $[2,13]$

$$
s \tilde{\hat{P}}(\mathbf{k}, s)-1=-\frac{|\mathbf{k}|^{\alpha} \phi}{\tau} \tilde{\hat{P}}(\mathbf{k}, s)
$$

which, in turn, is the Laplace-Fourier inverse of the spatial FFPE

$$
\partial_{t} P(\mathbf{x}, t)=\frac{\phi}{\tau}|\nabla|^{\alpha} P(\mathbf{x}, t) \text {. }
$$

The FFPE therefore exhibits a diverging mean square displacement, $\left\langle x^{2}\right\rangle[3]$. For this reason, an alternative rescaled form for the second moment holds, $\left\langle|x|^{\delta}\right\rangle \propto t^{\delta / \alpha}$, where $0<\delta<\alpha \leqslant 2$ when the diffusion is unbiased.

Analogously, for subdiffusive processes, it is the exponent in the waiting time distribution (with, in this instance, a diverging mean) that must be amended [2].

The derivation of the spatial FFPE from the CTRW, highlights the direct link between microscopic (jump and dwell time) distributions and $\alpha$, the anomalous exponent. In particular, spatial FFPEs make key predictions on the jump size distribution of random walkers $[9,18]$ that should be observed if individual particle trajectories are tracked [18-20]. And, furthermore, if the matter at hand is to understand the microscopic origin of $\alpha$, then the CTRW redirects our modeling attention to this important question [21]: what properties of the random walker's environment justify the divergent second moment of the jump size distribution?

Here we consider the case where individual particles are not tracked but a profile still appears to match a spatial FFPE. In this case, it is fair to ask whether other microscopic models could have described the evolution of this density equally well [22]. This brings us to the heart of our problem.

We will consider a concentration profile $c(\mathbf{x}, t)$ that evolves according to an apparent spatial FFPE (equation (6))

$$
\partial_{t} c(\mathbf{x}, t)=\gamma|\nabla|^{\alpha} c(\mathbf{x}, t)
$$


where $\gamma$ is a diffusion-like coefficient of the FFPE (with units of [position ${ }^{\alpha} /$ time]). Our focus will be on spatial FFPEs exhibiting superdiffusion which, for simplicity, we now simply refer to as FFPEs.

Contrary to other approaches from which the FFPE is derived [2], we will consider a regular Fokker-Planck equation (FPE) as an alternative [23]

$$
\partial_{t} c(\mathbf{x}, t)=-\nabla \cdot\left(\frac{\mathbf{F}(\mathbf{x}, t)}{\zeta} c(\mathbf{x}, t)\right)+D \nabla^{2} c(\mathbf{x}, t)
$$

where $\zeta$ is a friction coefficient and $D$ a normal diffusion coefficient. We will show that we can reproduce the behavior of the FFPE (equation (7)) using a regular FPE.

As a simple motivating example, consider a 1-d FFPE $(\alpha=1)$ that we can first solve analytically. For open boundary conditions and delta function initial conditions, the solution is [13]

$$
c(x, t)=\frac{1}{\pi} \cdot \frac{\gamma t}{\gamma^{2} t^{2}+x^{2}} .
$$

Substituting this expression for $c(x, t)$ back into equation (8) and solving for the force $F$ we obtain

$$
\frac{F(x, t)}{\zeta}=x\left(\frac{1}{t}-\frac{2 D}{x^{2}+\gamma^{2} t^{2}}\right)
$$

Equation (10) confirms that for this simple example there exists a force in the FPE that generates the evolution of a density precisely matching that of an FFPE with $\alpha=1$. This simple example also illustrates that this effective force depends explicitly both on time and space as well as the normal diffusion coefficient $D$. We add that since $D$ and $\zeta$ (through $F$ ) are chosen independently, the system may not satisfy the fluctuation-dissipation theorem. In addition, since the force initially varies sharply with time (as is the case in equation (10)), it may lie outside the linear-response regime.

In general, for problems not tractable analytically, we turn to numerics and ask: what spatially and temporally varying effective forces, $\mathbf{F}(\mathbf{x}, t)$, generate concentration profiles indistinguishable from those obtained from an FFPE (equation (7)) for a given set of initial conditions and diffusion coefficient $D$ ? Since we will rely on numerics, we will be limited to extracting forces from regions of profiles where the concentration is not vanishingly small.

Throughout our manuscript, we will assume that the diffusion coefficient would be a given quantity either estimated theoretically from the Stokes-Einstein relation [23] or determined from a priori control experiments.

In what follows, we tackle the inverse problem of using profiles generated from FFPEs to recover the spatially and time-dependent effective forces that could have given rise to the profiles otherwise described by FFPEs. Such inverse modeling methods are poised to yield quantitative insight into the dynamics of complex biological systems [24]. We will discuss how our effective force's time and spatial dependence may yield microscopic insight into some biophysical processes. 


\section{Materials and methods}

Our goal is to find the force in the regular FPE (equation (8)) that best describes an apparent anomalous diffusion concentration profile's evolution obtained from the solution of the FFPE (equation (7)) given a set of initial conditions and a diffusion coefficient, $D$.

To solve this problem, we first generate a concentration profile (which we call $\bar{c}(\mathbf{x}, t)$ ) by solving equation (7) on a grid (in space and time) according to the prescription provided by [25]. We treat this synthetic profile as an example of anomalous data. In particular, we take the discretizable Riemann-Liouville definition of the fractional derivative [25]

$$
\begin{aligned}
& \partial_{x}^{\alpha} c(\mathbf{x}, t) \\
& \equiv \frac{1}{\Gamma(n-\alpha)} \partial_{x}^{n} \int_{a}^{x} \frac{\mathrm{d} \xi}{(x-\xi)^{\alpha-n+1}} \cdot c(\xi, y, z, t) \\
& +\frac{(-)^{n}}{\Gamma(n-\alpha)} \partial_{x}^{n} \int_{x}^{b} \frac{\mathrm{d} \xi}{(\xi-x)^{\alpha-n+1}} \cdot c(\xi, y, z, t)
\end{aligned}
$$

where $n$ is an integer $(0 \leqslant n-1<\alpha<n)$. Since we consider cases where $1 \leqslant \alpha<2$, we take $n=2$. Equivalent expressions hold for the $y$ and $z$ spatial directions while $[a, b]$ define $x$ 's domain. For simplicity we assume diffusion symmetric in space; the generalization to an asymmetric process is straightforward [25]. As an aside, for conservative forces, these forces can be converted into a potential. For instance, for the heavy tailed distribution generated from the superdiffusive FFPE in one dimension, we expect a force derived from an unstable (i.e. inverted) potential that changes in time.

To find the force (or more precisely $\mathbf{F} / \zeta$ ) from the profile $\bar{c}(\mathbf{x}, t)$, we define a $\chi^{2}$ misfit statistic as follows

$$
\chi^{2}(t)=\int \mathrm{d} \mathbf{x}\left(\partial_{t} \bar{c}(\mathbf{x}, t)+\nabla \cdot\left(\frac{\mathbf{F}(\mathbf{x}, t)}{\zeta} \bar{c}(\mathbf{x}, t)\right)-D \nabla^{2} \bar{c}(\mathbf{x}, t)\right)^{2}
$$

where - for discrete data - all derivatives are interpreted as finite differences and integrals as sums. The integral is over all space (in practice, the region over which the bulk of the density of $\bar{c}(\mathbf{x}, t)$ lies) and $D$, as mentioned earlier, is a known specified quantity.

For simplicity only, we assume open spatial boundary conditions and follow the recipe provided by [25] to discretize and numerically solve our FFPE.

We then use conjugate gradient methods to minimize our $\chi^{2}$ (code available under publications on statphysbio.physics.iupui.edu) with respect to the force at each position for a given time and obtain a force, or $\mathbf{F}(\mathbf{x}, t) / \zeta$, valid over that time slice. Next, from the concentration profile at some later time $\left(t^{\prime}>t\right)$, we minimize $\chi^{2}\left(t^{\prime}\right)$ again, and find $\mathbf{F}\left(\mathbf{x}, t^{\prime}\right) / \zeta$.

In practice, for rough profiles, extracting the force requires some regularization of the $\chi^{2}$ minimization [24]. What follows is an illustration - a proof of principle - on smooth concentration profiles.

Our recipe is not specific to any type of boundary conditions, dimension or $\alpha$ (provided $1 \leqslant \alpha<2)$ 


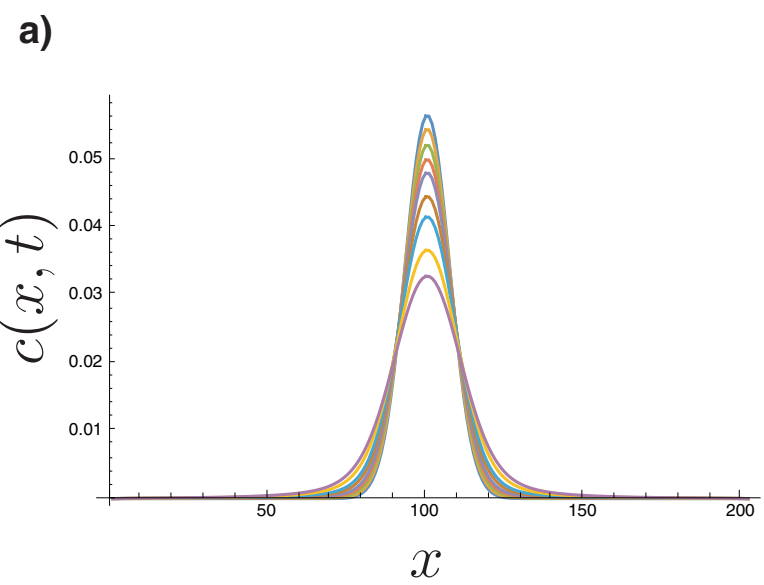

b)

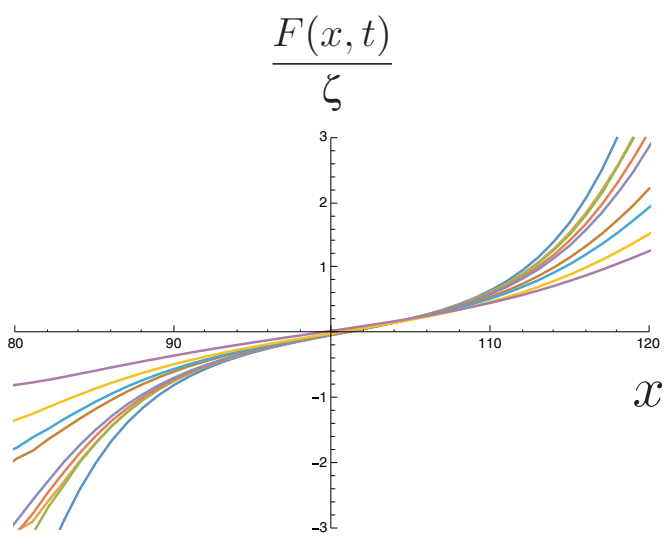

Figure 1. Effective forces (shown in b) are extracted from FFPE profiles (shown in a). We first evolve a Gaussian profile (our initial condition) with an the FFPE $(\alpha=1.2)$. Our initial profile (starting at time $\delta t=1)$ is shown as the sharpest curve in $(a)$. Later profiles $(\delta t=5,10,15,20,30,40,60,80)$ are increasingly broad. In $(b)$ we show forces extracted from those same time points (with the most nonlinear force reflecting the earliest time point and forces for future time-points becoming increasingly linear). Parameters used: $D=5, \Delta x=1, \delta t=0.06$, $\gamma=5, \alpha=1.2$. $D$ has units of $\left[\right.$ position $^{2} /$ time $], \gamma$ has units of $\left[\right.$ position $^{\alpha} /$ time $]$.

\section{Results and discussion}

We begin with an initial Gaussian concentration profile (in 1-d for simplicity only) and simulate its time evolution according to the FFPE. Figure 1(a) shows the evolution of a profile for $\alpha=1.2$ with broader profiles representing later time points; see details in caption. In real experiments, these data could represent fluorescence line scans repeated over one region to detect the profile of labeled proteins diffusing in a live cell.

From these profiles at different time points, we extract forces using equation (12) that would give rise to the heavy-tailed profile generated from the solution of the FFPE. The forces - shown in figure 1(b) - show a strong deviation from the linear force expected from an inverted harmonic potential in one-dimension.

Since forces are extracted by comparing concentration profiles at different points in space and time (equation (12)), we cannot accurately extract forces in regions of space where the concentration profile $\bar{c}(\mathbf{x}, t)$ is low. In other words, in regions where the concentration profile is low any force could be used to evolve that density since, by equation (8), this force would be multiplying a numerically vanishingly small number. The fact that forces far out in the tails cannot be accurately captured is a shortcoming of numerical methods. However, to our benefit, such forces - in regions where $\bar{c}(\mathbf{x}, t)$ is low-are largely irrelevant to the evolution of those regions where the majority of the density lies (which, as we will see, still exhibit a typical non-Gaussian form).

Thus in figure $1(b)$, forces away from $x=0$ and closer to the tails of the distribution, $x=80$ and $x=120$, show increased numerical errors (that is, deviation away from the symmetry expected). Figure 1(b) also shows that, initially, forces show greater variation with time than forces extracted for later times. As a consequence, a force evaluated at 


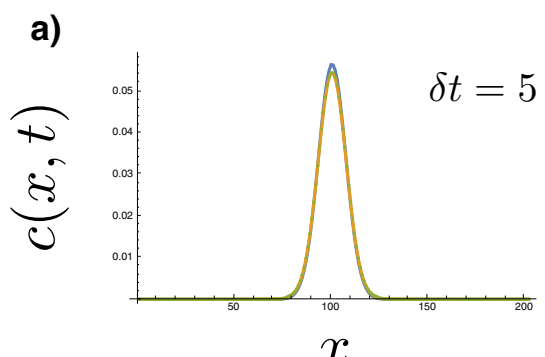

$x$

b)

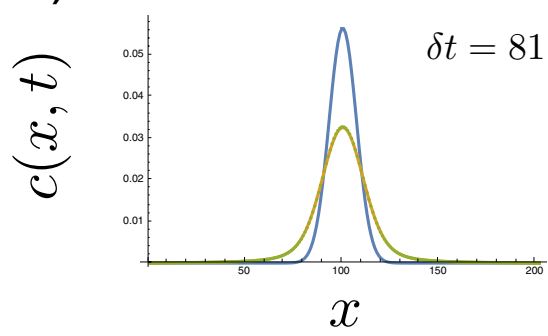

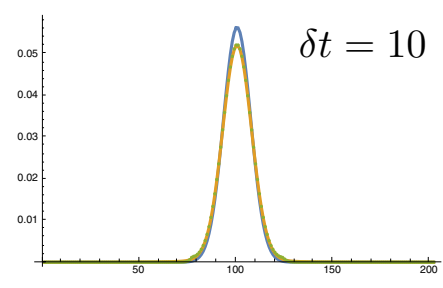

$x$

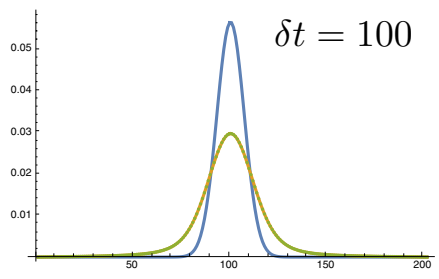

$x$

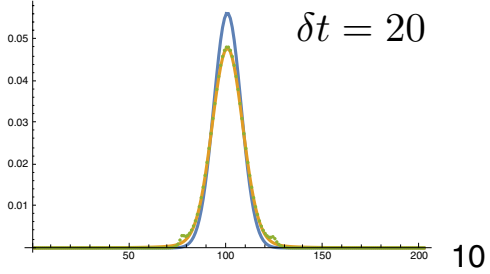

$x$

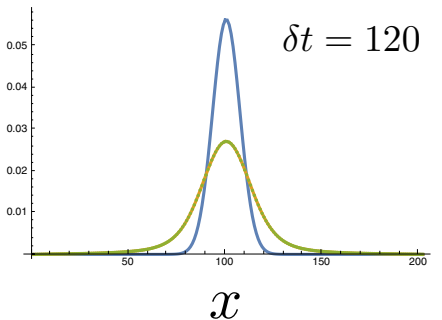

Figure 2. Forces evaluated at specific time points can predict the evolution of the profile over some future time interval. In general for forces evaluated at later time points (where forces vary more slowly with time), the time interval over which they can reproduce the evolution of the profile that matches the solution to the FFPE lengthens. In $(a)$ we show the time evolution of the profile (dotted line) evolved according to the force extracted at $\delta t=1$ (force originally shown in figure $1(b)$ ). The profile generated according to this force no longer matches the shape of the theoretical profile (exact solution to the FFPE, solid line) near $\delta t=20$. The initial condition, blue profile, is shown in blue on all plots. Analogously in $(b)$ we show the profile evolved from the force extracted at $\delta t=80$ (dotted line) at various later time points. The force still accurately predicts the evolution of the profile $40 \delta t$ later (as determined by how well the theoretical solution to the FFPE, solid line, and the profile evolved according to the force at $\delta t=80$ still match at $\delta t=120$ ). (We use the same parameters as in figure 1).

an early time point may only allow the profile to be evolved over a short period of time before the profile begins deviating from the solution to the FFPE. By contrast, forces evaluated at later time points can predict the evolution of the profile over a longer period of time. This is quantitatively shown in figure 2 .

While predicting the evolution of concentration profiles using forces extracted from the data serves to benchmark our method, our goal is not to regenerate profiles already known from the data. Rather our goal, as we will describe in greater depth later, is to derive insight from forces extracted at different time points.

Nonetheless, it is still worth asking about the minimal number of force time 'slices' required to regenerate the data. Briefly, here we show that even one force slice may suffice over a broad time interval. To do so, we extract a phenomenological force averaged over 
a)

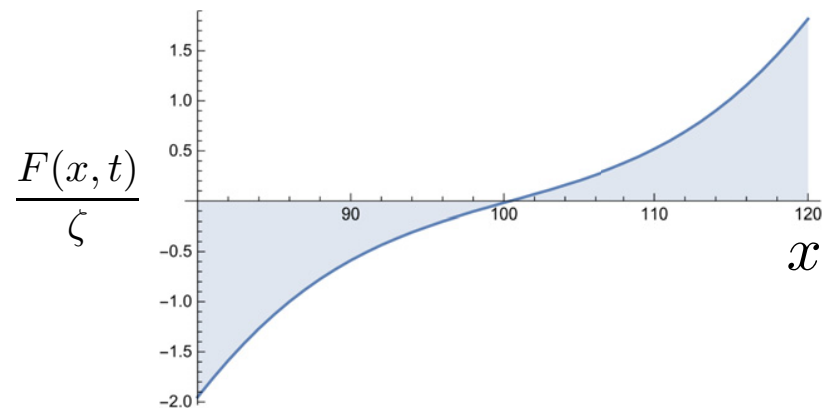

b)

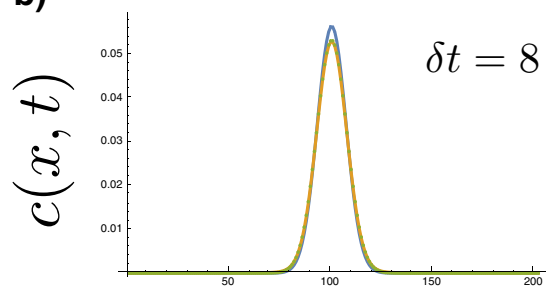

$x$

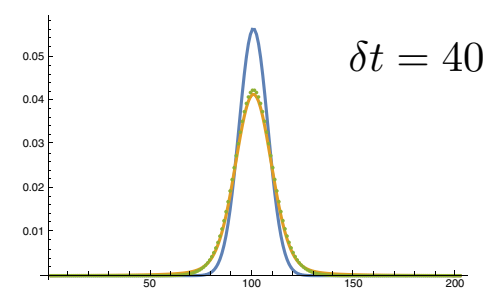

$x$

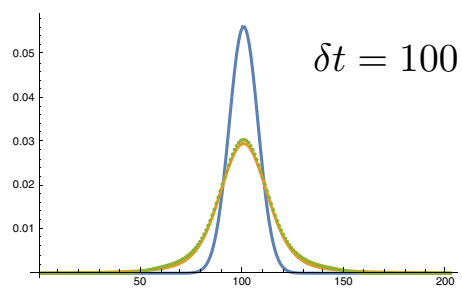

$x$

Figure 3. An average force predicts the evolution of a concentration profile over a long time interval. We began by extracting an average force, shown in $(a)$, over $100 \delta t$ using equation (13). As can be seen in (b), this phenomenological force reliably predicts the evolution of the profile over a time interval longer than the exact forces extracted at specific time points (figure 2). We use the same parameters used in the figures above.

all time points. This force is extracted from the data by minimizing

$$
\chi^{2}=\int \mathrm{d} \mathbf{x} \mathrm{d} t\left(\partial_{t} \bar{c}(\mathbf{x}, t)+\nabla \cdot\left(\frac{\mathbf{F}(\mathbf{x}, t)}{\zeta} \bar{c}(\mathbf{x}, t)\right)-D \nabla^{2} \bar{c}(\mathbf{x}, t)\right)^{2}
$$

In practice, the integral over time is over all time points for which a profile is available.

Initially, we used equation (13) to obtain an approximate time-averaged force (figure $3(a)$ ) from over 100 slices (from $\delta t=1$ to $\delta t=100$ ) of a profile evolved according to an FFPE with $\alpha=1.2$ (see details in caption). The initial profile we used is shown as the blue profile in figure $3(b)$. We then used the approximate force we extracted to evolve this initial profile (dotted lines in figure $3(b)$ ) to compare to the exact profile evolved according to the FFPE (solid lines in figure 3(b)). Figure 3(b) illustrates the predictive power of even one approximate force over a long time interval.

We now investigate the sensitivity of forces to changes in $\alpha$. Rather than to compare forces for two different $\alpha$ 's at multiple time points, for convenience we compare a single effective force: the approximate (time-averaged) force for both profiles. Figure 4(a) shows two profiles evolved from the an initial condition (blue curve in figure $4(a)$ ) according to $\alpha=1.2$ (broadest curve in figure $4(a)$ ) and $\alpha=1.8$ (remaining curve in figure $4(a)$ ) for $\delta t=180$; see details in caption. Figure $4(b)$ shows the approximate forces extracted. From this, we argue that we can detect differences in forces extracted from profiles generated from FFPEs using different $\alpha$ 's.

Here is the type of mechanistic insight that can be obtained from forces extracted from the evolution of FFPE's. Suppose we monitor profiles of a labeled protein $X$ by collecting line scans across a region of a live cell, as is routinely done to collect concentration profile 

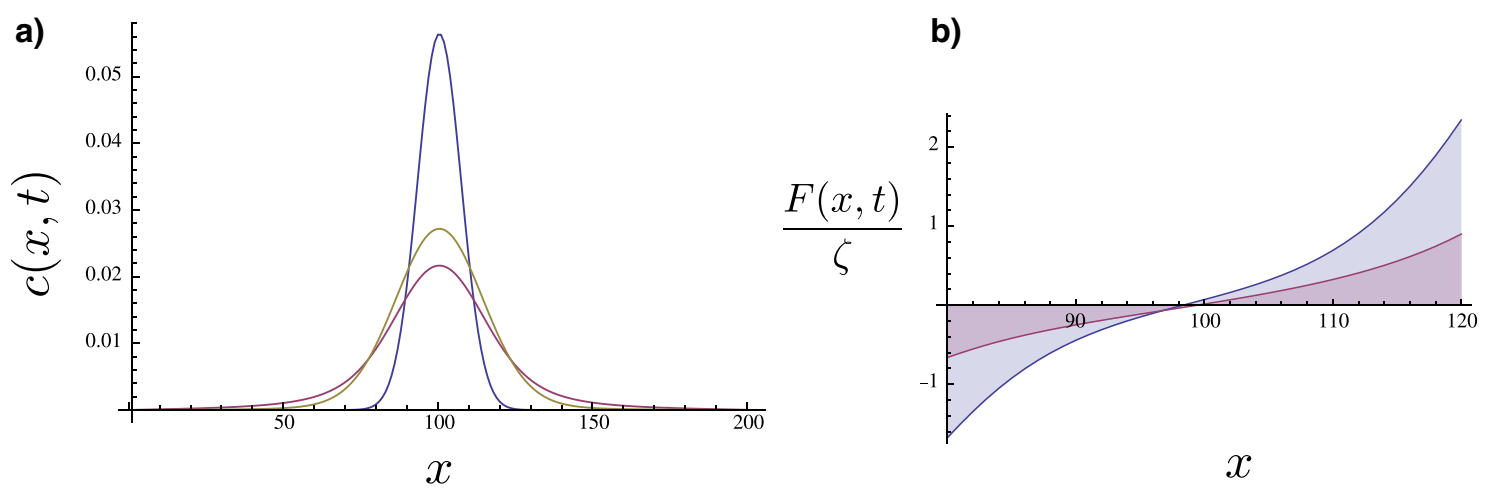

Figure 4. A hypothetical protein responsible for the transport of protein $X$ is mutated and we ask: how does the effective force acting on $X$ change? The sharpest peak in $(a)$ denotes the Gaussian initial concentration profile. The other profiles are solutions to the FFPE with $\alpha=1.2$ (red curve) and $\alpha=1.8$ (yellow curve); $\gamma=5$ in both cases. Just to be clear, unlike other plots, these are all theoretical curves. The average effective forces required to regenerate solutions to the FFPE are shown in (b). The more non-linear force (blue) coincides with the force required to generate a profile of the FFPE with $\alpha=1.2$, the other curve coincides with the force required to generate a profile of the FFPE with $\alpha=1.8$. If upon mutating $X$ 's binding partner the anomalous exponent changes from $\alpha=1.2$ to $\alpha=1.8$, we instead can use our method to find regions over which $X$ 's binding partner was applying a force on $X$. See text for details.

changes with high temporal resolution [26]. A protein $Y$ - known to assist in the transport and recruitment of $X$ to the outer cell membrane - is mutated and can no longer bind $X$. We suppose that the best fit exponent in the FFPE - which models $X$ 's profile changes in space and time - rises from $\alpha=1.2$ to $\alpha=1.8$ suggesting a transition towards a normal diffusion process for $X$ upon mutation of $Y$; see figure 4. By contrast to fitting the data with an FFPE, extracting the forces from the evolution of $X$ 's profile reveals the precise difference in driving force along distinct regions of the cell; see figure 4. In particular, this observation predicts the region of the cell where our hypothetical protein $Y$ is most active and, in doing so, may also identify regions where a driving force is present even in the absence of $Y$.

We mentioned earlier that we were extracting forces given known initial conditions and a fixed $D$ in our FPE. We end with final remarks on this point:

(1) Initial conditions. The force we extract from a concentration profile depends on the initial conditions. For instance, the force extracted from a profile evolved according to the FFPE with a sharp Gaussian distribution as its initial condition is different from the force extracted from a profile evolved from an initial mixture of two sharp Gaussian distributions centered at different values. Put differently, if the anomalous diffusion is driven by an effective force, then the anomalous exponent $\alpha$ depends on initial conditions.

(2) Diffusion coefficients. We made a choice to fix the diffusion coefficient in the regular FPE, equation (8), to some value motivated by a Stokes-Einstein form, say, and subsequently use the FFPE profile to find the effective force in the FPE. In future 
work, it would be interesting to explore the possibility of distributions of spatially and time-dependent diffusion coefficients [27].

\section{Conclusion}

We have shown that inverse methods - which have previously been used to extract underlying forces in cells [28] as well as diffusion coefficients and transition rates [29] from single particle tracking data-may provide principled strategies to resolve mechanistic insight from anomalous diffusion data.

We have explored one alternative to the FFPE and shown how inverse methods may be used to infer forces in an FPE that could otherwise give rise to profiles generated using FFPEs. In some cases, the effective forces we extract may help draw mechanistic insight into the origin of anomalous diffusion.

We emphasize that our method is not specific to any one force. While we focused on extracting effective forces that are required to recapitulate profiles generated by FFPEs, we could have explored different forces and some forces may be more biologically relevant than others.

For instance, a force's spatial dependence may tell us about local crowding in different cellular environments [30,31] or suggest the types of cellular structures interacting with our random walkers at specific locations [32]. Thus, future - more detailed-experiments could seek specific interaction partners responsible for a force change at a specific point in space.

Next, an effective force's time dependence may arise for a number of biological reasons: (1) on slower timescales, the remodeling of cytoskeletal structures (the biological 'highway' along which our random walkers may superdiffuse) [10, 33-35]; while on faster timescales, (2) ATP depletion or excesses [33]; and, perhaps most excitingly, (3) the degree of metabolic activity in the cell which has very recently been linked to the fluidity of the bacterial cytoplasm responsible for untrapping cellular structures [36]. Thus, understanding how a force depends on time (and, in particular, the timescale over which a force varies) may suggest what phenomena are responsible for the anomalous underlying dynamics.

The goal of theoretical biophysics is, ultimately, to generate quantitative and predictive biological models for complex phenomena from first principles [24,37]. Quantifying effective forces that identify changes as they occur within the cell from the data is one step along this path.

\section{Acknowledgments}

SP acknowledges the NSF MCB Grant Award No. 1412259 and his Start-up from IUPUI. SP also acknowledges both referees for their helpful suggestions. 


\section{References}

[1] Ghosh K, Dill K, Inamdar M, Seitaridou E and Phillips R 2006 Teaching the principles of statistical dynamics Am. J. Phys. 74123

[2] Metzler R and Nonnenmacher T F 2002 Space- and time-fractional diffusion and wave equations, fractional Fokker-Planck equations, and physical motivation Chem. Phys. 28467

[3] Metzler R and Klafter J 2000 The random walk's guide to anomalous diffusion: a fractional dynamics approach Phys. Rep. 33977

[4] Banks D S and Fradin C 2005 Anomalous diffusion of proteins due to molecular crowding Biophys. J. 89 2960

[5] Caspi A, Granek R and Elbaum M 2000 Enhanced diffusion in active intracellular transport Phys. Rev. Lett. 855655

[6] Wachsmuth M, Waldeck W and Langowski J 2000 Anomalous diffusion of fluorescent probes inside living cell nuclei investigated by spatially-resolved fluorescence correlation spectroscopy J. Mol. Biol. 298677

[7] Gal N and Weihs D 2010 Experimental evidence of strong anomalous diffusion in living cells Phys. Rev. E 81020903

[8] Hoefling F and Franosch T 2012 Anomalous transport in the crowded world of biological cells Rep. Prog. Phys. 76046602

[9] Dybiec B 2010 Anomalous diffusion on finite intervals J. Stat. Mech. P01011

[10] Tolić-Nørrelykke I V, Munteanu E L, Thon G, Oddershede L and Berg-Sørensen K 2004 Anomalous diffusion in living yeast cells Phys. Rev. Lett. 93078102

[11] Bancaud A, Huet S, Daigle N, Mozziconacci J, Beaudouin J and Ellenberg J 2009 Molecular crowding affects diffusion and binding of nuclear proteins in heterochromatin and reveals the fractal organization of chromatin EMBO J. 283785

[12] Klafter J, Shlesinger M F and Zumofen G 1996 Beyond Brownian motion Phys. Today 4933

[13] Jespersen S, Metzler R and Fogedby H C 1999 Lévy flights in external force fields: Langevin and fractional Fokker-Planck equations and their solutions Phys. Rev. E 592736

[14] Magdziarz M, Weron A, Burnecki K and Klafter J 2009 Fractional Brownian motion versus the continuoustime random walk: a simple test for subdiffusive dynamics Phys. Rev. Lett. 103180602

[15] Kneller G R and Hinsen K 2004 Fractional Brownian dynamics in proteins J. Chem. Phys. 12110278

[16] Mandelbrot B B and Van Ness J W 1967 Fractional Brownian motions, fractional noises and applications SIAM Rev. 10422

[17] Montroll E W and Weiss G H 1965 Random walks on lattices. II J. Math. Phys. 6167

[18] Wong I Y, Gardel M L, Reichman D R, Weeks E R, Valentine M T, Bausch A R and Weitz D A 2004 Anomalous diffusion probes microstructure dynamics of entangled F-actin networks Phys. Rev. Lett. 92 178101

[19] Jeon J H, Tejedor V, Burov S, Barkai E, Selhuber-Unkel C, Berg-Sørensen K, Oddershede L and Metzler R 2011 In vivo anomalous diffusion and weak ergodicity breaking of lipid granules Phys. Rev. Lett. 106 048103

[20] Saxton M J and Jacobson K 1997 Single-particle tracking: applications to membrane dynamics Ann. Rev. Biophys. Biomol. Struct. 26373

[21] Ott A, Bouchaud J P, Langevin D and Urbach W 1990 Anomalous diffusion in 'living polymers': a genuine Levy flight? Phys. Rev. Lett. 652201

[22] Gunawardena J 2014 Models in biology: accurate descriptions of our pathetic thinking BMC Biol. 1229

[23] Zwanzig R 2001 Nonequilibrium Statistical Mechanics (Oxford: Oxford University Press)

[24] Pressé S, Ghosh K, Lee J and Dill K A 2013 Principles of maximum entropy and maximum caliber in statistical physics Rev. Mod. Phys. 851115

[25] Liu F, Anh V and Turner I 2004 Numerical solution of the space fractional Fokker-Planck equation J. Comp. Appl. Math. 166209

[26] Rubart M 2004 Two-photon microscopy of cells and tissue Circ. Res. 951154

[27] Sengupta P, Garai K, Balaji J, Periasamy N and Maiti S 2003 Measuring size distribution in highly heterogeneous systems with fluorescence correlation spectroscopy Biophys. J. 841977

[28] Masson J-B, Casanova D, Türkcan S, Voisinne G, Popoff M R, Vergassola M and Alexandrou A 2009 Inferring maps of forces inside cell membrane microdomains Phys. Rev. Lett. 102048103

[29] Persson F, Lindén M, Unoson C and Elf J 2013 Extracting intracellular diffusion states and transition rates from single-molecule tracking data Nat. Meth. 10265 
[30] Woringer M, Darzacq X and Izeddin I 2014 Geometry of the nucleus: a perspective on gene expression regulation Curr. Opin. Chem. Biol. 20112

[31] Serag M F, Abadi M and Habuchi S 2014 Single-molecule diffusion and conformational dynamics by spatial integration of temporal fluctuations Nat. Commun. 55123

[32] Jankevics H, Prummer M, Izewska P, Pick H, Leufgen K and Vogel H 2005 Diffusion-time distribution analysis reveals characteristic ligand-dependent interaction patterns of nuclear receptors in living cells Biochem. 44 11676

[33] Bursac P, Lenormand G, Fabry B, Oliver M, Weitz D A, Viasnoff V, Butler J P and Fredberg J J 2005 Cytoskeletal remodelling and slow dynamics in the living cell Nat. Mater. 4557

[34] Zhou C, Slaughter B D, Unruh J R, Eldakak A, Rubinstein B and Li R 2011 Motility and segregation of hsp104-associated protein aggregates in budding yeast Cell 1471186

[35] Gourlay C W and Ayscough K R 2005 The actin cytoskeleton in ageing and apoptosis FEMS Yeast Res. 5 1193

[36] Parry B R, Surovtsev I V, Cabeen M T, O'Hern C S, Dufresne E R and Jacobs-Wagner C 2014 The bacterial cytoplasm has glass-like properties and is fluidized by metabolic activity Cell 156183

[37] Wang J, Xu L, Xue K and Wang E 2008 Exploring the origin of power law distribution in single-molecule conformation dynamics: energy landscape perspectives Chem. Phys. Lett. 463405 